\title{
Mitofusin-2 is a novel anti-angiogenic factor in pancreatic cancer
}

\author{
Zhichuan Lin ${ }^{1}$, Xiaoyi Lin ${ }^{1}$, Jinhong Chen ${ }^{1}$, Guoqiang Huang ${ }^{1}$, Tangen Chen ${ }^{1}$, Liling Zheng ${ }^{2}$ \\ ${ }^{1}$ Department of Hepatobiliary Surgery, Zhangzhou Affiliated Hospital, Fujian Medical University, Zhangzhou, China; ${ }^{2}$ Pediatric Intensive Care Unit, \\ Zhangzhou Affiliated Hospital, Fujian Medical University, Zhangzhou, China \\ Contributions: (I) Conception and design: Z Lin, X Lin, L Zheng; (II) Administrative support: J Chen, G Huang; (III) Provision of study materials or \\ patients: G Huang, T Chen; (IV) Collection and assembly of data: Z Lin, X Lin, J Chen, G Huang, L Zheng; (V) Data analysis and interpretation: Z \\ Lin, X Lin; (VI) Manuscript writing: All authors; (VII) Final approval of manuscript: All authors. \\ Correspondence to: Liling Zheng. Pediatric Intensive Care Unit, Zhangzhou Affiliated Hospital, Fujian Medical University, 59 Shengli West Road, \\ Xiangcheng District, Zhangzhou, China. Email: li5247522@163.com.
}

\begin{abstract}
Background: Aberrant expression of mitofusin-2 (MFN2) has been found to be associated with vascular endothelial growth factor A (VEGFA)-mediated angiogenesis in human umbilical vein endothelial cells (HUVECs). This study aimed to investigate the expression of MFN2 in pancreatic cancer (PC) and the role of MFN2 in vascular endothelial cell growth and angiogenesis.

Methods: Protein and mRNA expression of MFN2 and VEGFA were measured. The CCK-8 assay, tube formation assay, flow cytometry, and transmission electron microscopy were used to examine the effects of MFN2 overexpression on HUVEC growth, angiogenesis, and apoptosis. Western blot and immunocytochemical staining were conducted to measure alterations in cell cycle and apoptosis regulators and vascular endothelial growth factor receptor 2 (VEGFR2), angiopoietin-1 gene (ANGPT1), and tissue inhibitor of metalloproteinase 1 (TIMP1) expression in HUVECs.

Results: The results showed that MFN2 levels were significantly decreased in tumor tissues. Contrasting results were observed for VEGFA mRNA levels. MFN2 overexpression inhibited cell growth while promoting the formation of apoptotic bodies in HUVECs. Additionally, MFN2 overexpression enhanced the protein expression of p21 and p27 while attenuating the expression of proliferating cell nuclear antigen, VEGFA, VEGFR2, ANGPT1, and TIPM1 in HUVECs.
\end{abstract}

Conclusions: In conclusion, MFN2 expression negatively correlates with VEGFA expression in PC and inhibits endothelial cell growth and angiogenesis.

Keywords: Pancreatic cancer (PC); mitofusin-2 (MFN2); vascular endothelial growth factor A (VEGFA); angiogenesis

Submitted Mar 05, 2021. Accepted for publication Apr 17, 2021.

doi: 10.21037/jgo-21-176

View this article at: http://dx.doi.org/10.21037/jgo-21-176

\section{Introduction}

Pancreatic cancer (PC) represents the $11^{\text {th }}$ most common cancer and the $7^{\text {th }}$ leading cause of cancer death worldwide, with over 400,000 new cases and 400,000 deaths in 2018 (1). It has been projected that $\mathrm{PC}$ will become the $2^{\text {nd }}$ leading cause of cancer death by 2030 (2). Surgical management remains the mainstay of treatment for PC. However, $80-90 \%$ of PC patients are diagnosed at an advanced stage with unresectable tumors (3). Therefore, it is necessary to develop novel diagnostic markers and therapeutic approaches for PC.

Angiogenesis refers to the process of blood vessel formation from preexisting vessels, playing an essential role in the growth and metastasis of many tumors, including PC (4). Angiogenesis occurs when pro-angiogenic factors predominate over anti-angiogenic factors (5). Vascular endothelial growth factor A (VEGFA) is the most often described and critical pro-angiogenic factor that exerts 
mitogenic and anti-apoptotic effects on vascular endothelial cells (6). VEGFA promotes endothelial cell proliferation, migration, and tube formation primarily via the interaction with vascular endothelial growth factor receptor 2 (VEGFR2) (7). Targeting VEGFA/VEGFR2 signaling has become an established therapeutic strategy for many types of tumors $(8,9)$. However, the clinical outcomes of anti-angiogenic therapy remain poor for PC patients (10-12). Identifying new therapeutic targets may enhance the efficacy of anti-angiogenic treatment in PC.

Many studies have proven that mitochondrial cascades in vascular endothelial cells control angiogenesis by regulating endothelial cell proliferation, migration, and survival (13). Mitofusin-2 (MFN2), also named hyperplasia suppressor gene, is a transmembrane GTPase mainly expressed in the mitochondrial outer membrane. MFN2 plays a critical role in mitochondrial dynamics, mitochondrial bioenergetics, and cell fate decision (14). Additionally, MFN2 acts as a suppressor of cell proliferation in different cell models and is considered an anti-tumor gene in cancer $(2,15,16)$. Downregulated MFN2 has been observed in many cancers, such as colon cancer, liver cancer, and lung cancer (16-18). However, the function of MFN2 in vascular endothelial cells and tumors remains unclear. A previous study demonstrated that VEGFA upregulates MFN2 expression in human umbilical vein endothelial cells (HUVECs), and knockdown of MFN2 suppresses VEGFA-induced HUVEC migration and differentiation, suggesting an involvement of MFN2/VEGFA interaction in endothelial cells during angiogenesis (19). Most of the previous studies focused on the regulating role of VEGFA in the PC. But little known about how to effectively regulate VEGFA via MFN2. Therefore, we hypothesized that MFN2 interacts with VEGFA to regulate endothelial cell proliferation, playing an important role in angiogenesis during PC progression. These may be an innovation regulation mode for VEGFA in PC.

To test this hypothesis, we examined the expression patterns of MFN2 and VEGFA in PC tissues, and explored the effects of MFN2 overexpression on cell growth and survival in cultured HUVECs. Furthermore, we detected the alterations of cell cycle-related proteins and VEGFR2 in response to MFN2 overexpression. Our findings suggest that MFN2 might be a novel anti-angiogenic factor in PC. We present the following article in accordance with the MDAR reporting checklist (available at http://dx.doi. org/10.21037/jgo-21-176).

\section{Methods}

\section{Patients}

Pancreatic tumor tissue and paracancer tissue samples $(>5 \mathrm{~cm}$ from the tumor margin) were collected from 52 patients with primary PC who had undergone pancreaticoduodenectomy at Zhangzhou Hospital Affiliated to Fujian Medical University (Zhangzhou, Fujian, China) from January 2014 to January 2015. The clinical characteristics of the patients are summarized in Table 1. The criteria for TNM staging was based on the new WHO classification (19). All tissue samples were immediately processed after surgery. Diagnosis, grading, and staging were confirmed independently by two pathologists in blinding. This study was approved by the Ethics Committee of Fujian Medical University. Each patient provided written consent. All procedures performed in this study involving human participants were in accordance with the Declaration of Helsinki (as revised in 2013).

\section{Cell culture}

HUVECs and 293T cells were obtained from the Cell Bank of the Chinese Academy of Sciences (Shanghai, China) and grown in Dulbecco's modified Eagle medium supplemented with $10 \%$ fetal bovine serum and $1 \%$ penicillin/ streptomycin in a humidified atmosphere of $5 \% \mathrm{CO}_{2}$.

\section{Construction of MFN2-overexpressing adenoviral vectors and transfection}

Human MFN2 cDNA was subcloned into the HindIII/ KpnI site of an adenoviral vector Ad5GFP (Invitrogen, Carlsbad, CA, USA). The recombinant adenoviral vector was transfected into $293 \mathrm{~T}$ cells using Lipofectamine 2000 (Thermo Fisher Scientific, Waltham, MA, USA) to package virus particles expressing MFN2 (Ad5MFN2). The adenovirus was purified by gradient density ultracentrifugation. HUVECs were transfected with Ad5MFN2 or the negative control Ad5GFP. Green fluorescence was visualized $24 \mathrm{~h}$ after transfection under a fluorescence microscope (Nikon, Japan, provided by Fujian Medical University, Zhangzhou, Fujian, China).

\section{Cell growth assay}

HUVECs were plated on sterile slides in a 6-well plate 
Table 1 The mRNA levels of MFN2 and VEGFA in pancreatic cancer tissue samples from patients with pancreatic cancer

\begin{tabular}{|c|c|c|c|c|c|c|c|}
\hline Characteristics & $\mathrm{n}$ & \multicolumn{3}{|c|}{ MFN2 } & \multicolumn{3}{|c|}{ VEGFA } \\
\hline Gender & & & 0.969 & 0.337 & & 0.852 & 0.398 \\
\hline Male & 27 & $0.3870 \pm 0.0587$ & & & $0.8865 \pm 0.0594$ & & \\
\hline Female & 25 & $0.3710 \pm 0.0604$ & & & $0.0599 \pm 0.0119$ & & \\
\hline$<60$ & 12 & $0.3849 \pm 0.0557$ & & & $0.8579 \pm 0.0659$ & & \\
\hline$\geq 60$ & 40 & $0.3775 \pm 0.0612$ & & & $0.9050 \pm 0.0529$ & & \\
\hline Tumor diameter $(\mathrm{cm})$ & & & 1.842 & 0.071 & & 4.511 & 0.0001 \\
\hline$<5$ & 32 & $0.3921 \pm 0.0647$ & & & $0.8681 \pm 0.6336$ & & \\
\hline$T_{1-2}$ & 42 & $0.3905 \pm 0.0588$ & & & $0.8786 \pm 0.0563$ & & \\
\hline$T_{3-4}$ & 10 & $0.3327 \pm 0.0357$ & & & $0.9548 \pm 0.0194$ & & \\
\hline $\mathrm{N}$ stage & & & 1.215 & 0.23 & & 5.366 & 0.0001 \\
\hline $\mathrm{N}_{0}$ & 27 & $0.3697 \pm 0.0518$ & & & $0.8596 \pm 0.0618$ & & \\
\hline$N_{1-3}$ & 25 & $0.3897 \pm 0.0663$ & & & $0.9296 \pm 0.0267$ & & \\
\hline M stage & & & 1.905 & 0.063 & & 7.798 & 0.0001 \\
\hline$M_{0}$ & 42 & $0.3849 \pm 0.0602$ & & & $0.8839 \pm 0.0566$ & & \\
\hline $\mathrm{M}_{1}$ & 10 & $0.3369 \pm 0.0322$ & & & $0.9649 \pm 0.0151$ & & \\
\hline II & 28 & $0.3896 \pm 0.0602$ & & & $0.9139 \pm 0.0170$ & & \\
\hline III & 7 & $0.3872 \pm 0.0833$ & & & $0.9190 \pm 0.0299$ & & \\
\hline IV & 5 & $0.3369 \pm 0.0595$ & & & $0.9649 \pm 0.0151$ & & \\
\hline
\end{tabular}

MFN2, mitofusin-2; VEGFA, vascular endothelial growth factor A.

at a density of $1 \times 10^{5}$ cells/well. Cells were transfected with Ad5MFN2 or Ad5GFP and incubated for $48 \mathrm{~h}$. After washing with phosphate-buffered saline (PBS), cells were fixed with $4 \%$ paraformaldehyde and stained with hematoxylin. Cells were then washed with tap water and stained with eosin. After rinsing with tap water, the slides were dried and sealed with neutral resin. Images were acquired under a CK-40 inverted microscope (Olympus, Tokyo, Japan).

\section{Quantitative real-time polymerase chain reaction (qRT-PCR)}

Total RNA was isolated using Trizol reagent (Invitrogen), according to the manufacturer's instructions. One microgram of RNA was reverse-transcribed into cDNA using the EnergicScript ${ }^{\circledR}$ first strand cDNA synthesis kit (ShineGene Molecular Biotech, Shanghai, China), according to the manufacturer's protocol. Amplification 
was performed using the ShineSybr ${ }^{\circledR}$ real time qPCR kit (ShineGene Molecular Biotech) and gene-specific primers (MFN2, forward: 5'-GTGCTCAACGCCAGGATTC-3', reverse: 5'-CGAACCGCCTCTGCAATC-3'; VEGFA, forward: 5'-CGAACCGCCTCTGCAATC-3', reverse: 5'-TGTTGGACTCCTCAGTGGGC-3'; GAPDH, forward: 5'-ATCCCATCACCATCTTCCAGG-3', reverse: 5'-TGATGACCCTTTTGGCTCCC-3') on a qRTPCR device (Applied Biosystems, Foster City, CA, USA). GAPDH was used as an internal control.

\section{Western blot analysis}

HUVECs or pancreatic tissue samples were lysed or homogenized using lysis buffer on ice. The lysates were centrifuged at 12,000 rpm for $15 \mathrm{~min}$. Proteins in the lysates were quantified using a Bradford protein quantification kit (Sangon Biotech, Shanghai, China). Protein samples $(20 \mu \mathrm{g})$ were separated on a $10 \%$ SDS gel and transferred to a polyvinylidene fluoride membrane (Sangon Biotech). After blocking with $5 \%$ skim milk for $1 \mathrm{~h}$, the membrane was incubated with anti-MFN2 (1:1,000; Cell Signaling Technology, Danvers, MA, USA), anti-VEGFA (1:1,000; Sangon Biotech), anti-P21 (1:1,000; Ruiyingbio, Suzhou, Jiangsu, China), anti-P27 (1:1,000; Ruiyingbio), antiproliferating cell nuclear antigen (PCNA; 1:1,000; Ruiyingbio), anti-Bcl2 (1:1,000; Abcam, Cambridge, UK), anti-Caspase 3 (1:1,000, Abcam), anti-ANGPT1 (1:1,000; Abcam), anti-TIMP1 (1:1,000; Abcam), or anti- $\beta$-actin (1:1,000; Abcam) overnight at $4{ }^{\circ} \mathrm{C}$. After washing with Tris-buffered saline containing $0.1 \%$ Tween 20 (TBST), the membrane was incubated with horseradish peroxidase (HRP)-conjugated secondary antibody (1:10,000; Sangon Biotech) for $1 \mathrm{~h}$ at room temperature, followed by an additional 3 washes with TBST. The protein bands were detected using an enhanced chemiluminescence reagent (Sangon Biotech) and quantified using Quantity One software (Bio-Rad, Hercules, CA, USA). $\beta$-actin was used as an internal control.

\section{Immunocytochemical staining}

HUVECs were plated on sterile slides in a 6-well plate at a density of $1 \times 10^{5}$ cells/well. Cells were transfected with Ad5MFN2 or Ad5GFP and incubated for $48 \mathrm{~h}$. After washing with PBS, cells were fixed with $4 \%$ paraformaldehyde for $20 \mathrm{~min}$ at room temperature, followed by citric acid-mediated antigen retrieval. After blocking with $10 \%$ normal goat serum for $20 \mathrm{~min}$, cells were incubated with anti-VEGFR2 (1:100; Abcam) at $4{ }^{\circ} \mathrm{C}$ overnight, followed by incubation with HRPconjugated secondary antibody (Abcam) for $20 \mathrm{~min}$ at room temperature and hematoxylin counterstaining. After rinsing with tap water, the slides were dried and sealed with neutral resin. Images were acquired under the CK-40 inverted microscope.

\section{Transmission electron microscopy}

HUVECs were plated on sterile slides in a 6-well plate at a density of $1 \times 10^{5}$ cells/well. Cells were transfected with Ad5MFN2 or Ad5GFP and incubated for $48 \mathrm{~h}$. After washing with PBS, cells were prefixed with $2.5 \%$ glutaraldehyde for $2 \mathrm{~h}$ and post-fixed with $1 \%$ osmic acid for an additional $2 \mathrm{~h}$ at $4{ }^{\circ} \mathrm{C}$. A gradient dehydration was performed using ethanol $(30 \%, 50 \%$, and $70 \%)$ and acetone $(80 \%, 90 \%, 95 \%$, and $100 \%)$ sequentially. The slides were then embedded in resin, stained with lead citrate, and observed under a transmission electron microscope (TECNAI-10; Philips, Amsterdam, Netherlands, provided by Fujian Medical University, Zhangzhou, Fujian, China).

\section{Tube formation assay}

A total of $5 \times 10^{3}$ HUVECs were seeded in 96-well plates, which were pre-coated with Matrigel (BD Biosciences, San Jose, CA, USA) extracellular matrix (ECM). The plate was then incubated at $37^{\circ} \mathrm{C}$ for $8 \mathrm{~h}$. Later, tube formation was examined with a white light microscope and analyzed with NIH Image J software.

\section{Flow cytometry for the detection of apoptosis}

The Annexin V-FITC Apoptosis Detection Kit (Beyotime, Wuhan, China) was used for cell apoptosis determination. Briefly, $1 \times 10^{5}$ HUVECs were collected and washed twice with PBS. Next, the cells were collected and resuspended in $200 \mu \mathrm{L}$ Annexin V-FITC Binding Buffer containing $5 \mu \mathrm{L}$ Annexin V-FITC, and $10 \mu \mathrm{L}$ PI was added. The cells were then incubated at room temperature for $30 \mathrm{~min}$ in the dark. Finally, $300 \mu \mathrm{L}$ PBS was added to cell suspensions, and the samples were immediately analyzed under flow cytometry (FACS can; Beckman Coulter, Fullerton, CA). Data were analyzed using FlowJo software (Treestar, San Carlos, CA). 


\section{CCK-8 assay}

The growth of HUVECs was determined using the Cell Counting Kit-8 (CCK-8, Beyotime) according to the manufacturer's instructions. HUVECs were plated at a density of $5 \times 10^{3}$ cells per well in 96-well plates. After a continuous incubation of $24,48,72$, and $96 \mathrm{~h}, 10 \mu \mathrm{L}$ of CCK-8 solution was added into each well. After an incubation in the dark for another $2 \mathrm{~h}$, the absorbance was measured at a wavelength of $450 \mathrm{~nm}$.

\section{Statistical analysis}

All the data are publicly available via contacting to corresponding author. Data are expressed as the mean \pm standard deviation. Statistical analysis was performed using SPSS software version 19.0 (SPSS, Chicago, IL, USA). A paired $t$-test was used to analyze the differences in MFN2 and VEGFA levels between cancer tissues and corresponding normal tissues. Student's $t$-test or one-way ANOVA analysis were used to compare means among two groups or more than two groups, respectively. Pearson's correlation coefficient analysis was used to analyze the correlations. Differences between categorical variables were compared using the $\chi^{2}$ test. $\mathrm{P}<0.05$ was considered statistically significant.

\section{Results}

\section{MFN2 was downregulated in PC tissues}

To explore the involvement of MFN2 and the relationship between MFN2 and VEGFA in PC, we detected mRNA and protein expression of both MFN2 and VEGFA in paired pancreatic tumor tissue and paracancer tissue samples. As shown in Figure 1A, the MFN2 mRNA level was significantly decreased in tumor tissue in PC patients. Moreover, we analyzed the association between MFN2 mRNA levels and clinical factors. It was found that a lower MFN2 level was significantly associated with higher TNM stages (Table 1). Contrastingly, a higher level of VEGFA mRNA was found in PC tissues when compared to adjacent normal tissues (Figure 1B,C), and PC patients with upregulated VEGFA had larger tumor volumes, higher TNM stages, and higher histologic grades $(\mathrm{P}<0.05$, Table 1). Interestingly, the Pearson analysis showed that MFN2 and VEGFA mRNA levels were negatively correlated (Figure 1D). In addition, we found both that MFN2 and VEGFA had a moderate expression level in normal pancreatic tissue, while in PC tissues, MFN2 was lowly expression and VEGFA was significantly enhanced (Figure 1E, data from The Human Protein Atlas, 200x). These results suggest that low expression of MFN2 might be associated with the formation and progression of $\mathrm{PC}$ via modulating VEGFA.

\section{Overexpression of MFN2 inbibited cell growth of HUVECs}

Considering the promotive role of VEGFA in endothelial cell proliferation (7), and the possible negative correlation between MFN2 and VEGFA in PC, we further explored whether MFN2 could inhibit HUVEC growth. We transduced HUVECs with adenoviral vectors overexpressing MFN2 and observed green fluorescence (as control, 100×) in almost all transduced cells, suggesting a high transduction efficiency of the prepared vectors (Figure $2 A, B$ ). We next examined the effect of MFN2 overexpression on cell growth using the CCK-8 assay and hematoxylin and eosin staining $(100 \times$ and $400 \times)$. The results showed that overexpression of MFN2 significantly reduced cell growth and attenuated the number of cells, and promoted cell shrinkage in HUVECs compared to the control group (Figure 2C,D). These findings suggests that overexpression of MFN2 suppresses cell growth and induces cell death in HUVECs.

\section{Overexpression of MFN2 induced cell apoptosis in HUVECS}

Next, we examined cell apoptosis in MFN2-overexpressing HUVECs. We found that compared with the Ad5GFP group, upregulating MFN2 led to an enhanced apoptosis rate of HUVECs (Figure $3 A$ ), a higher level of cleaved caspase 3, and a lower level of Bcl2 (Figure 3B). Using transmission electron microscopy, we observed condensed chromatin and apoptotic bodies in MFN2-overexpressing HUVECs (Figure 3C), but not in control cells (Figure 3C, 2 millionX). Taken together, these findings suggest that MFN2 induces cell apoptosis in HUVECs.

\section{Overexpression of MFN2 attenuated the expression of PCNA while enhancing the expression of $p 21$ and $p 27$ in HUVECS}

To investigate the mechanism of MFN2 in regulating cell growth and survival in HUVECs, we measured the protein 
A

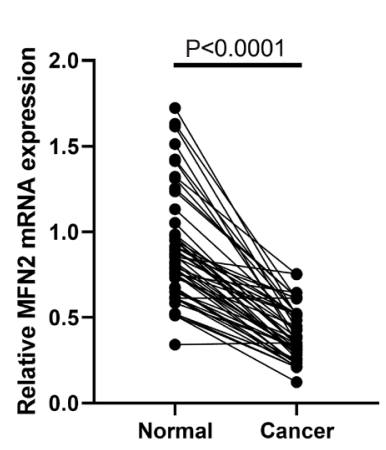

B

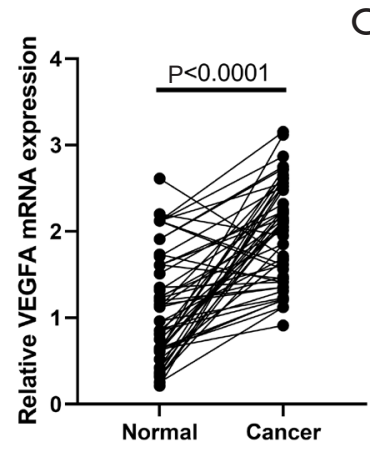

C

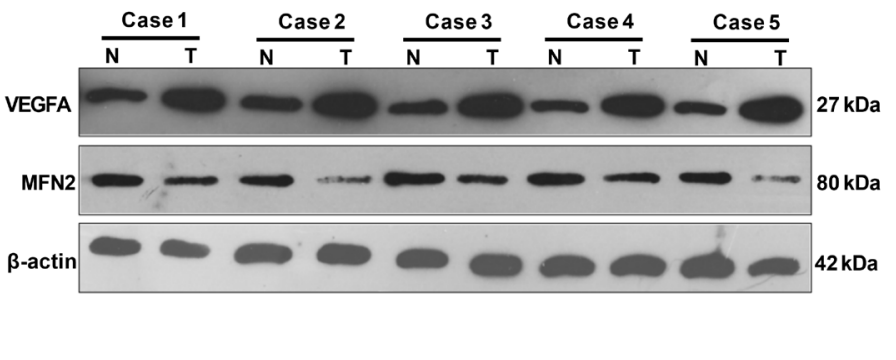

D

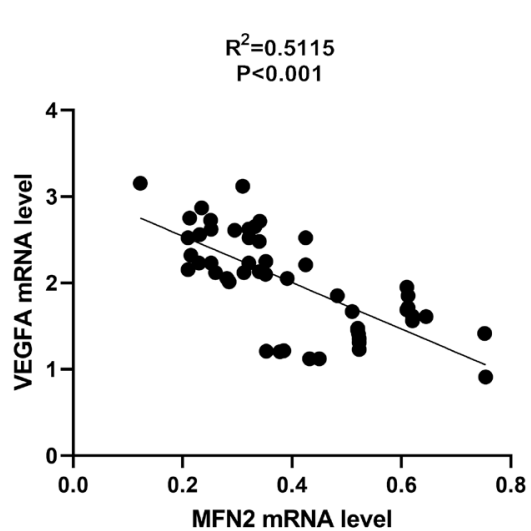

E

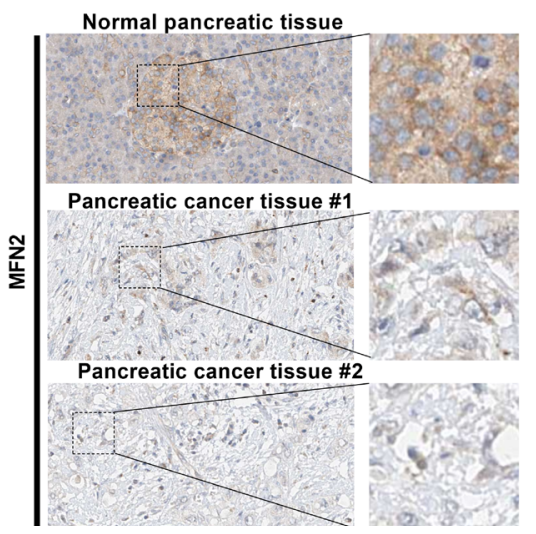

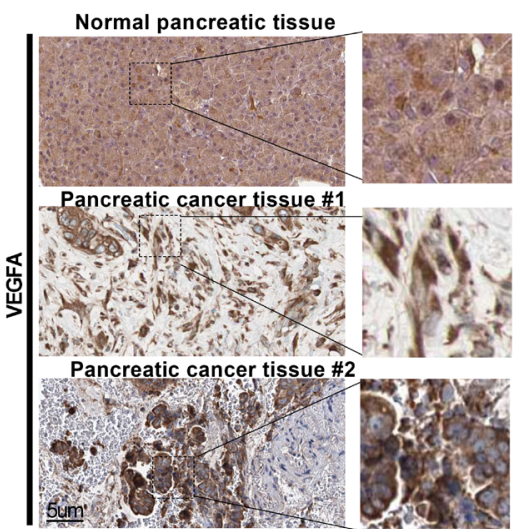

Figure 1 The expression patterns of MFN2 and VEGFA in pancreatic cancer. This study included 52 paired patients with primary PC. A and B. RT-PCR was used to detect MFN2 (A) and VEGFA (B) mRNA levels in the tumor tissues and adjacent normal tissues. (C) Western blot analysis was used to determine the protein expression of MFN2 and VEGFA in PC tissues and adjacent normal tissues. (D) Pearson analysis showed the correlations between MFN2 and VEGFA expression in PC tissues. (E) The IHC data of MFN2 and VEGFA expression in normal pancreatic tissues and 2 cases of PC tissues in the online database The Human Protein Atlas, Magnification 200×.

expression of PCNA as well as the G1 phase inhibitors p21 and p27. As shown in Figure 4, compared with controls, MFN2 overexpression remarkably enhanced the protein expression of $\mathrm{p} 21$ and $\mathrm{p} 27$, while attenuating the protein expression of PCNA in HUVECs. These findings suggest that MFN2 affects endothelial cell growth and survival possibly by inducing the imbalance of cell cycle regulators.

\section{MFN2 downregulated VEGFR2 expression in HUVECs}

We performed the tube formation assay to assess whether MFN2 could affect angiogenesis of HUVECs. It was found that when MFN2 was upregulated, the tube length of HUVECs reduced significantly (Figure $5 A$ ). As VEGFA promotes endothelial cell proliferation primarily through binding to VEGFR2 (7), we examined whether
MFN2 could regulate VEGFR2 and the expression of other angiogenic proteins (including ANGPT1 and TIMP1) in HUVECs. Immunocytochemical staining revealed a significant decrease in the protein expression of VEGFR2 in MFN2-overexpressing HUVECs (Figure 5B). Furthermore, the western blot results showed VEGFA, VEGFT2, ANGPT1, and TIMP1 protein expression levels were significantly repressed with MFN2 overexpression (Figure 5C). Collectively, these findings suggest that MFN2 regulates endothelial cell growth and survival possibly by blocking VEGFA/VEGFR2.

\section{Discussion}

Currently, the treatment options for pancreatic cancer are limited. This disease is usually diagnosed at a late stage, 
A

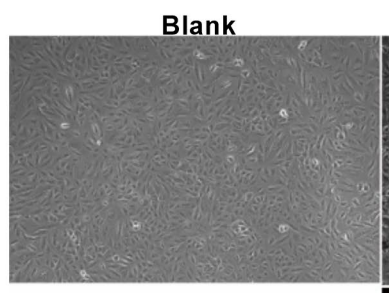

Ad5GFP

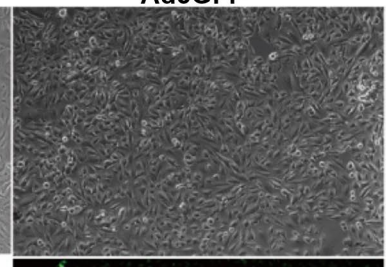

\section{GFP.}

Ad5MFN2

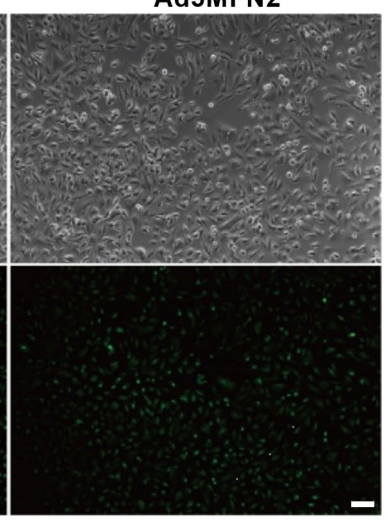

B

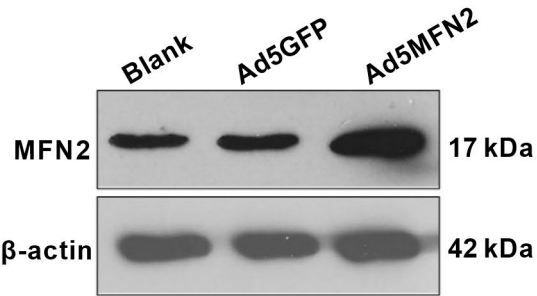

C

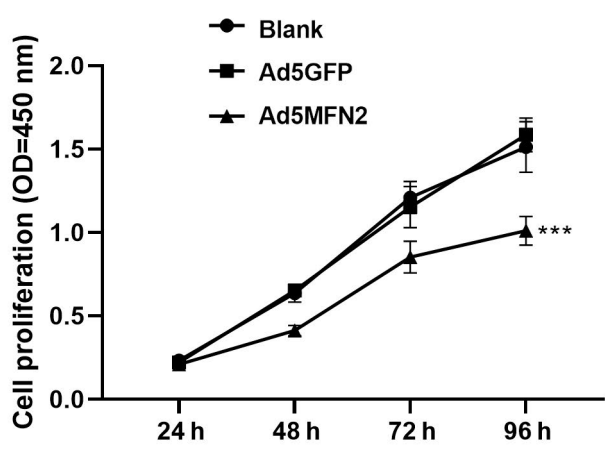

D

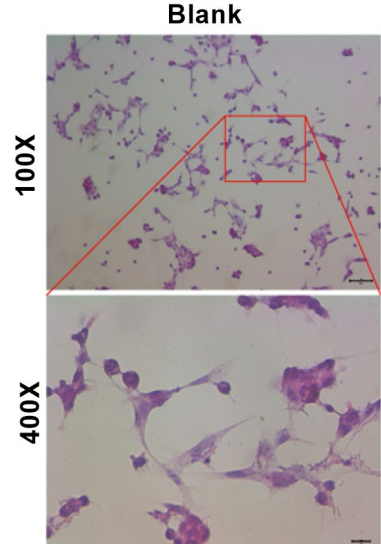

Ad5GFP

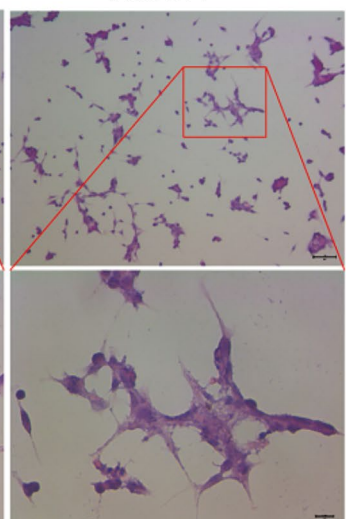

Ad5MFN2

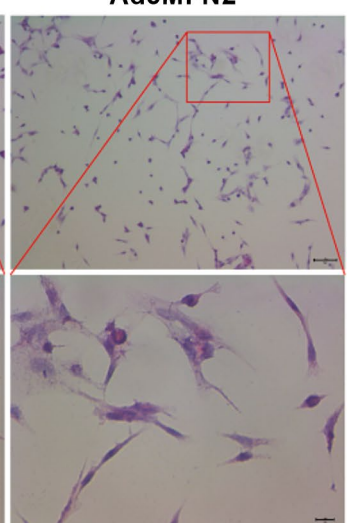

Figure 2 Overexpression of MFN2 inhibited cell growth and induced cell death in human umbilical vein endothelial cells (HUVECs). (A) Un-transfected HUVECs were used as a blank control. HUVECs were transfected with Ad5GFP or Ad5MFN2, and green fluorescence was observed $48 \mathrm{~h}$ after transfection under a fluorescence microscope. Magnification $100 \times$. (B) Western blot was used to detect MFN2 expression in HUVECs. (C) CCK-8 assay for the detection of cell growth. (D) The cells were fixed and stained with hematoxylin and eosin $48 \mathrm{~h}$ after transfection. Cells were observed under an inverted microscope. Representative images are shown. Magnification 100x, 400x. ***, $\mathrm{P}<0.001$ vs. Ad5GFP group. $\mathrm{N}=3$. Ad5MFN2, adenoviral vectors expressing human MFN2; Ad5GFP, adenoviral vectors expressing green fluorescent protein.

which prevents curative surgical resection. Chemotherapy is the most frequently used approach for pancreatic cancer treatment and has limited effects. Recently, the microenvironment formation and metastasis in PC has attracted a lot of attention, especially the angiogenesis. MFN2 acts as a tumor suppressor in many types of cancer via regulating angiogenesis $(20,21)$. However, the role of MFN2 in angiogenesis during cancer progression remains unclear. In the present study, we measured the mRNA levels of MFN2 and VEGFA in 52 pairs of primary pancreatic tumor tissue and paracancer tissue samples from PC patients. We demonstrated that MFN2 and VEGFA exhibited opposite expression patterns in PC tissues, suggesting that MFN2 and VEGFA might exert opposite effects in angiogenesis during cancer progression. We also evaluated the effects of MFN2 overexpression on cell growth and survival in HUVECs. Our results demonstrated that MFN2 inhibited cell growth while inducing apoptosis in HUVECs. Furthermore, we found that MFN2 overexpression significantly reduced the protein expression of PCNA while elevating the expression of p21 and p27, suggesting that MFN2 impacts cell fate by regulating cell cycle proteins. In addition, MFN2 overexpression resulted in a significant reduction in VEGFR2, ANGPT1, and TIMP1 expression in HUVECs, suggesting that MFN2 might suppress VEGFA/VEGFR2-mediated angiogenesis 
A
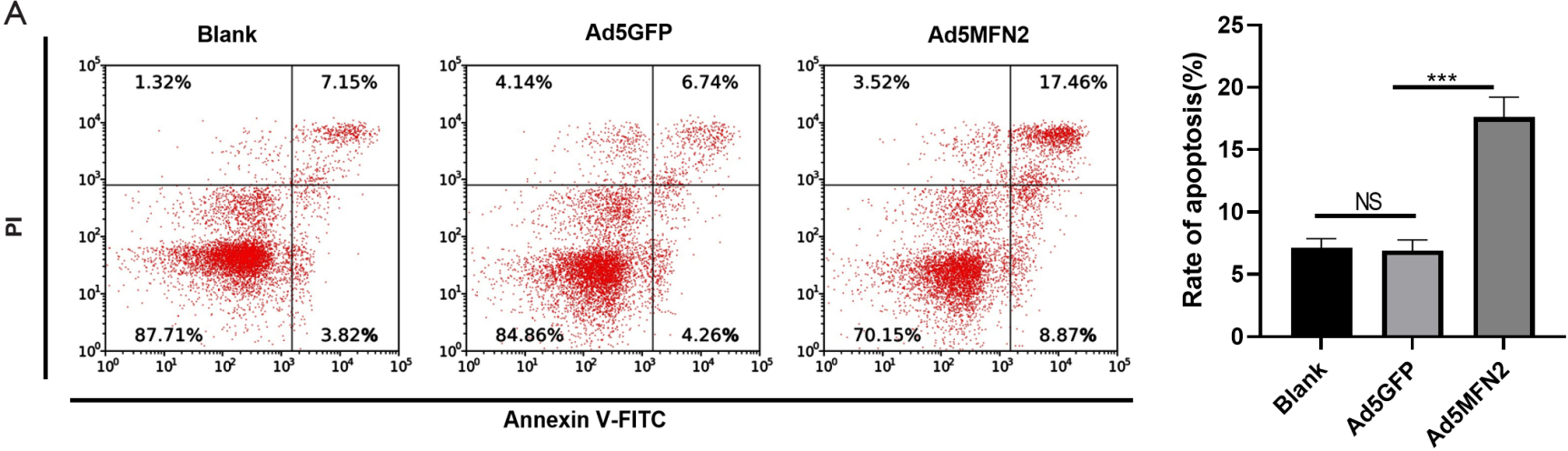

B
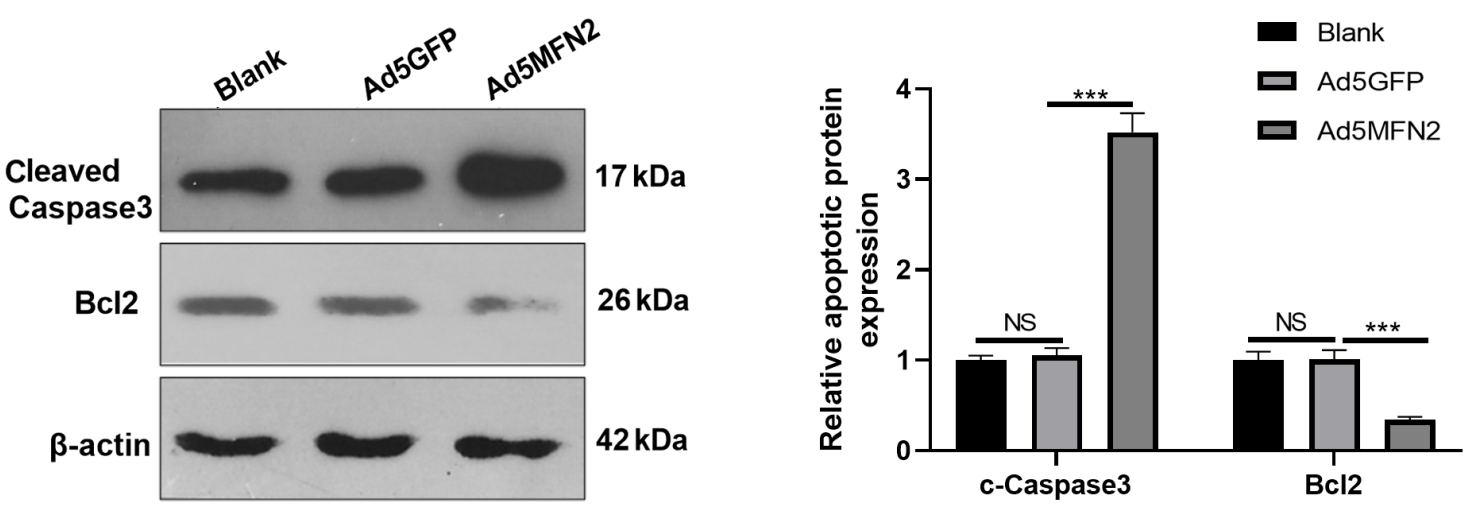

C

Blank

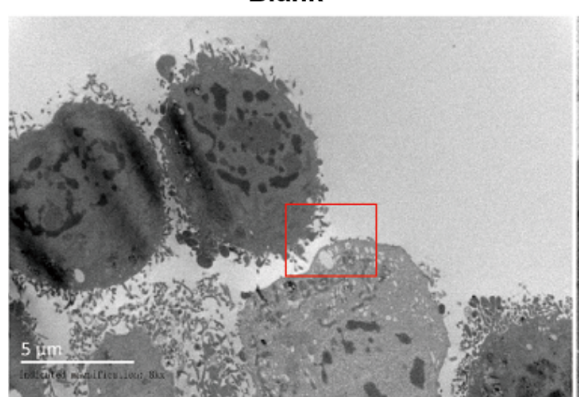

Ad5GFP

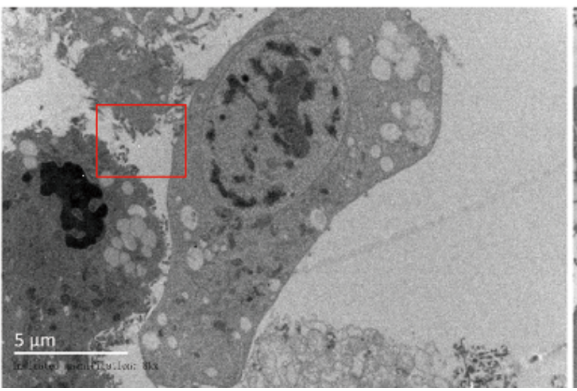

Ad5MFN2

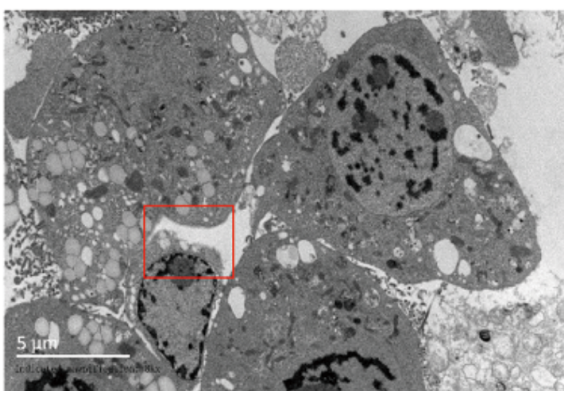

Figure 3 Overexpression of MFN2 induced cell apoptosis in HUVECs. (A) Flow cytometry was performed to evaluate cell apoptosis. (B) Western blot was used to detect caspase 3 and Bcl2 expression in HUVECs. (C) Ultrastructural changes and the formation of apoptotic bodies were observed under a transmission electron microscope $48 \mathrm{~h}$ after transfection. Representative images are shown. Scale bar $=5 \mu \mathrm{m}$. Ad5MFN2, adenoviral vectors expressing human MFN2; Ad5GFP, adenoviral vectors expressing green fluorescent protein. NS, P>0.05; ***, $\mathrm{P}<0.001 . \mathrm{N}=3$.

in endothelial cells. Our findings therefore identify MFN2 as a novel anti-angiogenic factor in PC.

Mammals have two mitofusins, namely MFN1 and MFN2, that catalyze outer mitochondrial membrane fusion via GTPase activity (22). Compared with MFN1, MFN2 is more frequently related to human diseases, including neurodegenerative diseases, cardiomyopathy, neuromuscular disorders, diabetes, and cancer (23-25). Although downregulation of MFN2 has been observed in various types of cancer (16-18), the expression of MFN2 in PC remains unexplored. Since MFN2 positively regulates mitochondrial fusion, and PC cells demonstrate increased 

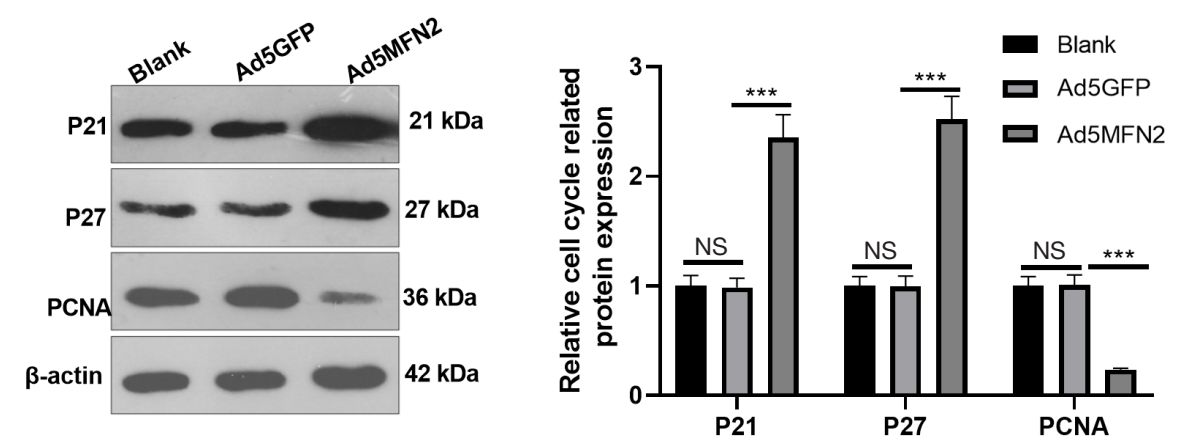

Figure 4 Overexpression of MFN2 affected the expression of cell cycle-related proteins. HUVECs were transfected with Ad5MFN2 or Ad5GFP. Un-transfected HUVECs were used as a blank control. Western blot analysis was performed $48 \mathrm{~h}$ after transfection to determine the protein expression of MFN2, PCNA, p21, and p27. $\beta$-actin was used as an internal control. NS, P>0.05; ${ }^{* *}, \mathrm{P}<0.001$. N=3. PCNA, proliferating cell nuclear antigen.

mitochondrial fragmentation (26), we speculated that MFN2 is downregulated in PC. In this study, we found that MFN2 mRNA levels were significantly decreased in tumor tissues from PC patients, especially those with higher $\mathrm{T}$ stages, suggesting that MFN2 is a potential biomarker in diagnosing PC.

VEGFA is essential for vasculogenesis and angiogenesis, as it regulates the activity and integrity of endothelial cells. During tumor angiogenesis, VEGFA acts as a potent angiogenic and survival factor for endothelial cells by promoting cell proliferation, migration, invasion, and vessel permeability via interacting with VEGFR2 (27). In human PC, VEGFA is commonly overexpressed and contributes to tumor growth and metastasis (28). On the other hand, MFN2 is involved in multiple cellular processes, such as endoplasmic reticulum-mitochondria junctions, axonal transport, and cell cycle progression $(14,29,30)$. In PC, MFN2 promotes cancer cell autophagy through suppressing the PI3K/Akt/mTOR pathway. MFN2 deficiency predicts poor prognosis in patients with PC (31). Pharmacologic activation of MFN2 significantly improved the median survival of mice with spontaneous PC by enhancing mitochondrial fusion (32), suggesting the therapeutic value of MFN2 in PC. Thus, we hypothesized that MFN2 might negatively regulate endothelial cell proliferation. Indeed, our results showed that overexpression of MFN2 inhibited cell growth, promoted apoptosis, and reduced angiogenesis in HUVECs, indicating the anti-angiogenic and proapoptotic roles of MFN2 in endothelial cells.

Considering the role of MFN2 in cell cycle progression, and to investigate whether MFN2 regulates cell cycle progression in HUVECs, we measured the levels of cell cycle-related proteins including PCNA, p21, and p27. The results indicated that overexpression of MFN2 remarkably attenuated the protein expression of p21 and p27, while enhancing the protein expression of PCNA. Consistently, studies have shown that MFN2 inhibits cell proliferation by upregulating p21 and p27 expression and/or downregulating PCNA expression in various cell models, including bladder, cervical, and breast cancer cells. Other cell cycle-related proteins, such as cyclin A and cyclin D1, are also involved in the regulatory effect of MFN2 on cell cycle progression (32-34). In addition, our results showed that MFN2 overexpression significantly reduced the protein expression of VEGFR2. Therefore, MFN2 might regulate endothelial cell growth and survival by inducing the imbalance of cell cycle regulators and blocking VEGFA/VEGFR2.

This study has some limitations. Firstly, we did not knock down the expression of MFN2 to evaluate whether it is essential for HUVEC growth and survival. Secondly, there is a lack of in vivo evidence to support our conclusions. Thirdly, the underlying mechanism of MFN2-induced alterations in cell cycle regulators and VEGFR2 remains unexplored.

In conclusion, we demonstrated that MFN2 is downregulated in high-grade PC, as well as in pancreatic tumor tissue. MFN2 potentially suppresses cell growth in HUVECs by inducing an imbalance in the expression of cell cycle regulators and by blocking VEGFA/VEGFR2, serving as a potential anti-angiogenic factor in PC progression. 
A

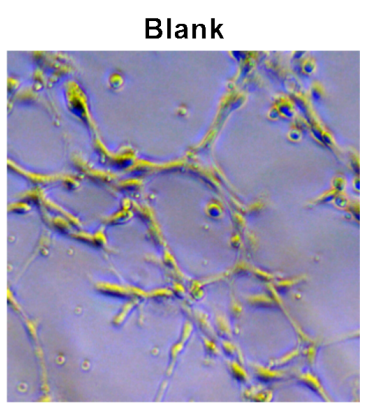

Ad5GFP

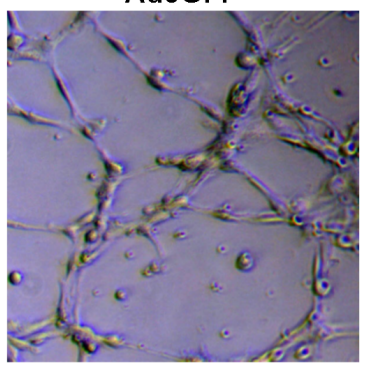

Ad5MFN2

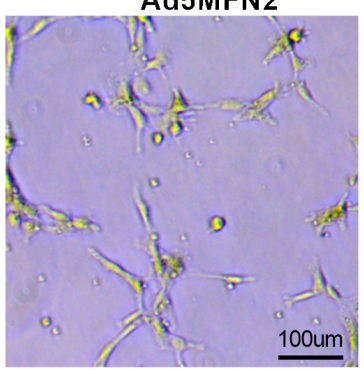

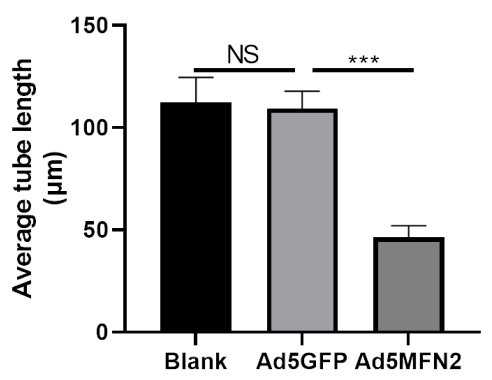

B
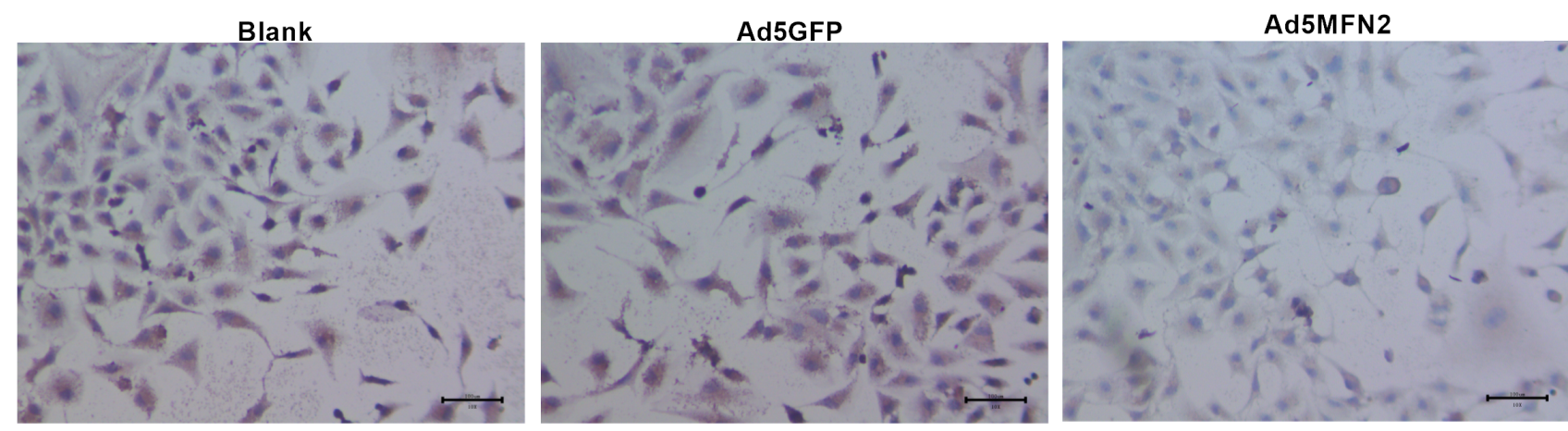

C
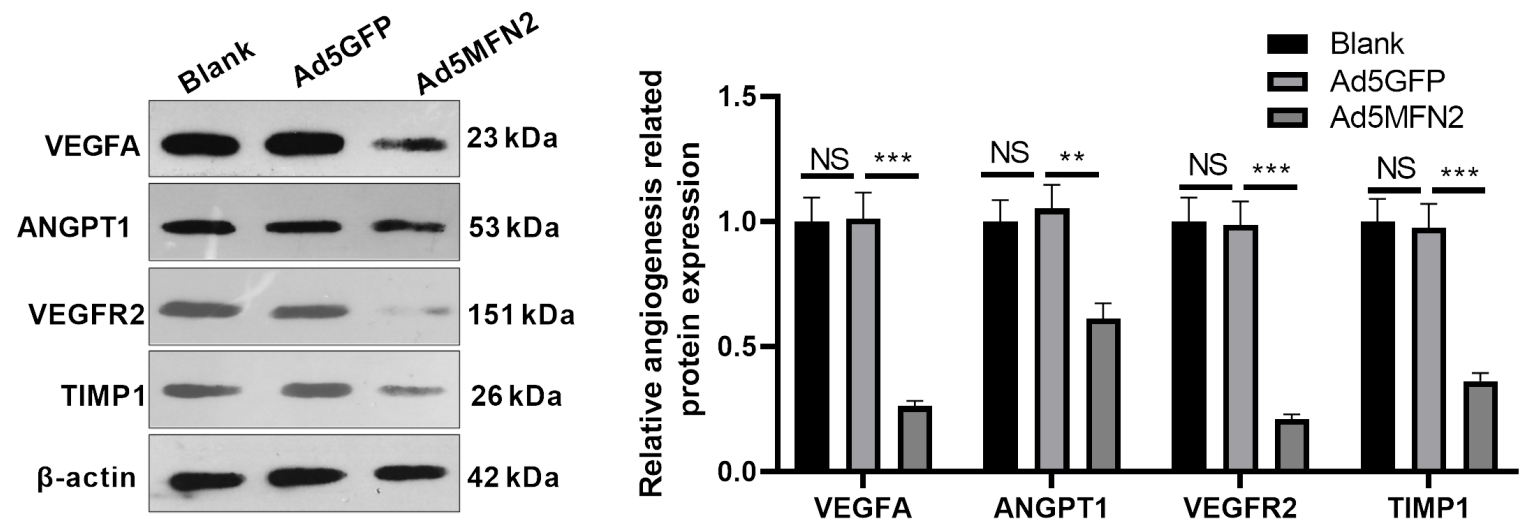

Figure 5 Overexpression of MFN2 inhibited angiogenesis and VEGFR2 expression in HUVECs. (A) The tube formation assay was used to evaluate the angiogenesis of HUVECs. (B) Immunocytochemical staining was performed $48 \mathrm{~h}$ after transfection to detect protein expression of VEGFR2 (brown staining) in HUVECs. Representative images are shown. Magnification 100×, 400×. (C) Western blot was carried out to determine VEGFA, VEGFT2, ANGPT1, and TIMP1 expression levels in HUVECs. NS, $\mathrm{P}>0.05 ;{ }^{* *}, \mathrm{P}<0.01 ;{ }^{* *}, \mathrm{P}<0.001 . \mathrm{N}=3$.

\section{Acknowledgments}

Funding: This work was supported by the Natural Science Foundation of Fujian, China (grant number 2017J01388).

\section{Footnote}

Reporting Checklist: The authors have completed the MDAR reporting checklist. Available at http://dx.doi.org/10.21037/ jgo-21-176

Data Sharing Statement: Available at http://dx.doi. org/10.21037/jgo-21-176

Conflicts of Interest: All authors have completed the ICMJE uniform disclosure form (available at http://dx.doi. org/10.21037/jgo-21-176). The authors have no conflicts of interest to declare. 
Ethical Statement: The authors are accountable for all aspects of the work in ensuring that questions related to the accuracy or integrity of any part of the work are appropriately investigated and resolved. This study was approved by the Ethics Committee of Fujian Medical University. Each patient provided written consent. All procedures performed in this study involving human participants were in accordance with the Declaration of Helsinki (as revised in 2013).

Open Access Statement: This is an Open Access article distributed in accordance with the Creative Commons Attribution-NonCommercial-NoDerivs 4.0 International License (CC BY-NC-ND 4.0), which permits the noncommercial replication and distribution of the article with the strict proviso that no changes or edits are made and the original work is properly cited (including links to both the formal publication through the relevant DOI and the license). See: https://creativecommons.org/licenses/by-nc-nd/4.0/.

\section{References}

1. Rawla P, Sunkara T, Gaduputi V. Epidemiology of Pancreatic Cancer: Global Trends, Etiology and Risk Factors. World J Oncol 2019;10:10-27.

2. Chen KH, Dasgupta A, Ding J, et al. Role of mitofusin 2 (Mfn2) in controlling cellular proliferation. FASEB J 2014;28:382-94.

3. Hidalgo M, Cascinu S, Kleeff J, et al Addressing the challenges of pancreatic cancer: future directions for improving outcomes. Pancreatology 2015;15:8-18.

4. Li S, Xu HX, Wu CT, Wang WQ, et al. Angiogenesis in pancreatic cancer: current research status and clinical implications. Angiogenesis 2019;22:15-36.

5. Baeriswyl V, Christofori G. The angiogenic switch in carcinogenesis. Semin Cancer Biol 2009;19:329-37.

6. Lal N, Puri K and Rodrigues B: Vascular Endothelial Growth Factor B and Its Signaling. Front Cardiovasc Med 2018;5:39.

7. Peach CJ, Mignone VW, Arruda MA, et al. Molecular Pharmacology of VEGF-A Isoforms: Binding and Signalling at VEGFR2. Int J Mol Sci 2018;19:1264.

8. Heath VL, Bicknell R. Anticancer strategies involving the vasculature. Nat Rev Clin Oncol 2009;6:395-404.

9. Ivy SP, Wick JY, Kaufman BM. An overview of smallmolecule inhibitors of VEGFR signaling. Nat Rev Clin Oncol 2009;6:569-79.

10. Yamaue $H$, Tsunoda $T$, Tani $M$, et al. Randomized phase
II/III clinical trial of elpamotide for patients with advanced pancreatic cancer: PEGASUS-PC Study. Cancer Sci 2015;106:883-90.

11. Rougier P, Riess H, Manges R, et al. Randomised, placebo-controlled, double-blind, parallel-group phase III study evaluating aflibercept in patients receiving firstline treatment with gemcitabine for metastatic pancreatic cancer. Eur J Cancer 2013;49:2633-42.

12. Gonçalves A, Gilabert M, Francois E, et al. BAYPAN study: a double-blind phase III randomized trial comparing gemcitabine plus sorafenib and gemcitabine plus placebo in patients with advanced pancreatic cancer. Ann Oncol 2012;23:2799-805.

13. Reichard A, Asosingh K. The role of mitochondria in angiogenesis. Mol Biol Rep 2019;46:1393-400.

14. Schrepfer E, Scorrano L. Mitofusins, from Mitochondria to Metabolism. Mol Cell 2016;61:683-94.

15. Chen KH, Guo X, Ma D, et al. Dysregulation of HSG triggers vascular proliferative disorders. Nat Cell Biol 2004;6:872-83.

16. Cheng X, Zhou D, Wei J, et al. Cell-cycle arrest at G2/ $M$ and proliferation inhibition by adenovirus-expressed mitofusin-2 gene in human colorectal cancer cell lines. Neoplasma 2013;60:620-6.

17. Wang $\mathrm{W}, \mathrm{Lu} \mathrm{J}, \mathrm{Zhu} F$, et al. Pro-apoptotic and antiproliferative effects of mitofusin-2 via Bax signaling in hepatocellular carcinoma cells. Med Oncol 2012;29:70-6.

18. Rehman J, Zhang HJ, Toth PT, et al. Inhibition of mitochondrial fission prevents cell cycle progression in lung cancer. FASEB J 2012;26:2175-86.

19. Lugus JJ, Ngoh GA, Bachschmid MM, et al. Mitofusins are required for angiogenic function and modulate different signaling pathways in cultured endothelial cells. J Mol Cell Cardiol 2011;51:885-93.

20. Li Y, Dong W, Shan X, et al. The anti-tumor effects of $\mathrm{Mfn} 2$ in breast cancer are dependent on promoter DNA methylation, the P21(Ras) motif and PKA phosphorylation site. Oncol Lett 2018;15:8011-8.

21. Xu K, Chen G, Li X, et al. MFN2 suppresses cancer progression through inhibition of mTORC2/Akt signaling. Sci Rep 2017;7:41718.

22. Ishihara N, Eura Y, Mihara K. Mitofusin 1 and 2 play distinct roles in mitochondrial fusion reactions via GTPase activity. J Cell Sci 2004;117:6535-46.

23. Züchner S, Mersiyanova IV, Muglia M, et al. Mutations in the mitochondrial GTPase mitofusin 2 cause CharcotMarie-Tooth neuropathy type 2A. Nat Genet 2004;36:44951. Erratum in: Nat Genet 2004;36:660. 
24. Wang X, Su B, Lee HG, et al. Impaired balance of mitochondrial fission and fusion in Alzheimer's disease. $\mathrm{J}$ Neurosci 2009;29:9090-103.

25. Filadi R, Pendin D, Pizzo P. Mitofusin 2: from functions to disease. Cell Death Dis 2018;9:330.

26. Anderson GR, Wardell SE, Cakir M, et al. Dysregulation of mitochondrial dynamics proteins are a targetable feature of human tumors. Nat Commun 2018;9:1677.

27. Koch S, Claesson-Welsh L. Signal transduction by vascular endothelial growth factor receptors. Cold Spring Harb Perspect Med 2012;2:a006502.

28. Tang RF, Wang SX, Peng L, et al. Expression of vascular endothelial growth factors $\mathrm{A}$ and $\mathrm{C}$ in human pancreatic cancer. World J Gastroenterol 2006;12:280-6.

29. Filadi R, Greotti E, Turacchio G, et al. Mitofusin 2 ablation increases endoplasmic reticulum-mitochondria coupling. Proc Natl Acad Sci U S A 2015;112:E2174-81.

30. Ngoh GA, Papanicolaou KN, Walsh K. Loss of mitofusin

Cite this article as: Lin Z, Lin X, Chen J, Huang G, Chen T, Zheng L. Mitofusin-2 is a novel anti-angiogenic factor in pancreatic cancer. J Gastrointest Oncol 2021;12(2):484-495. doi: 10.21037/jgo-21-176
2 promotes endoplasmic reticulum stress. J Biol Chem 2012;287:20321-32.

31. Xue R, Meng Q, Lu D, et al. Mitofusin2 Induces Cell Autophagy of Pancreatic Cancer through Inhibiting the PI3K/Akt/mTOR Signaling Pathway. Oxid Med Cell Longev 2018;2018:2798070.

32. Liu X, Sun J, Yuan P, et al. Mfn2 inhibits proliferation and cell-cycle in Hela cells via Ras-NF-kappaB signal pathway. Cancer Cell Int 2019;19:197.

33. Jin B, Fu G, Pan H, et al. Anti-tumour efficacy of mitofusin-2 in urinary bladder carcinoma. Med Oncol 2011;28 Suppl 1:S373-80.

34. Ma LI, Chang Y, Yu L, et al. Pro-apoptotic and antiproliferative effects of mitofusin-2 via PI3K/Akt signaling in breast cancer cells. Oncol Lett 2015;10:3816-22.

(English Language Editor: C. Betlazar-Maseh) 